\title{
Ergebnisse einer Reise nach dem Pacific (SCHAUINSLAND 1896-1897).
}

\section{Polycladen.}

\author{
Von \\ Dr. Marianne Plehn in München.
}

Mit 2 Abbildungen im Text.

Herr Prof. Dr. Schauinsland hat von seiner Reise nach Australien und der Südsee etwa 20 Polycladen mitgebracht, die er die Freundlichkeit hatte mir zur Untersuchung zu übergeben.

Die Thiere stammen grössten Theils von den $\mathrm{Ch}$ atham-Inseln und aus dem French Pass; ein Exemplar ausserdem aus Tasmanien, eines von der Insel Laysan. Die beiden letztern repräsentiren je eine besondere Form.

Die Polyclade aus Laysan ist ein Thysanozoon brocchii (GRUBe). Auf den Sandwich-Inseln, also in relativ geringer Entfernung von Laysan, ist bereits von Pease ${ }^{1}$ ) eine Polyclade gefunden worden, die er Peasia tentaculata nennt, die LANG aber auf Grund der Beschreibung der äussern Körperbeschaffenheit für identisch mit Thysanozoon brocchii hält, wie er überhaupt zahlreiche, von ältern Autoren schlecht beschriebene Thysanozoen dieser Species zuweist und dieselbe für kosmopolitisch erklärt. Strummer-Traunfels bestreitet die Berechtigung dieser Auffassung ${ }^{2}$ ) und will die Frage nach der $\mathrm{Zu}$ gehörigkeit jener zweifelhaften Arten mindestens offen lassen, speciell auch was Th. tentaculatum (Peasia tentaculata) betrifft. Das Th.

1) Citirt von A. Lang, Die Polycladen des Golfes von Neapel, in: Fauna Flora Neapel, Monogr. 9, Leipzig 1884.

2) Stummer-Traunfels, Tropische Polycladen. I. Das Genus Thysanozoon Grube, in: Arb. zool. Inst. Graz, V. 5, No. 4. 
rocchii von Laysan spricht nun aber gewiss zu Gunsten der LANGschen Ansicht von der weiten Verbreitung der Art.

Die Tasmanische Polyclade, die erste, die überhaupt von dort her bekannt wird, gehört in die Familie der Leptoplanidae, sie lässt sich aber nicht in eines der alten Genera einreihen. Da die Anordnung und Beschaffenheit der Augen eines der Merkmale ist, die am leichtesten $\mathrm{zu}$ constatiren und für die Leptoplaniden zur Unterscheidung der Genera besonders werthvoll sind, nenne ich das Thier, in Berücksichtigung seiner auffallend kleinen Augen, Microcelis und bezeichne die Species als

\section{Microcelis schauinstandi.}

Mein Exemplar ist von derber Consistenz; die Länge beträgt $25 \mathrm{~mm}$, die Breite $15 \mathrm{~mm}$. Genauere Angaben über die Form, z. B. ob die vordere oder die hintere Hälfte breiter ist, lassen sich nicht machen, da das Thier zu stark gefaltet und contrahirt ist. Ueberhaupt sind ja solche Angaben, wenn sie sich auf ein einziges, conservirtes Thier beziehen, stets von höchst fraglichem Werth. Der Rücken erscheint grünlich grau marmorirt; nach dem Rande zu wird die Farbe heller, ein schmaler Saum ist ganz farblos. Die Bauchseite ist ebenfalls gleichmässig weisslich. - Das ganze Vorderende des Thieres ist mit vielen Hunderten von Augen bedeckt; eine deutliche Gruppirung in Gehirnhof- und Tentakelaugen lässt sich nicht wahrnehmen; die sehr kleinen Augen sind gleichmässig vertheilt. Leider lässt der Erhaltungszustand des Thieres ein Studium des Baues dieser Augen nicht zu. Ausserdem enthält auch noch der Körperrand, mit Ausnahme seines hintern Viertels, eine Unmenge von Augen, die an Grösse den übrigen noch nachstehen. Auf der kleinen schematischen Skizze der Anatomie sind die Augen sämmtlich viel zu gross und lange nicht zahlreich genug angegeben; sie würden, selbst in der doppelten Vergrösserung der Skizze, nur mit einer guten Lupe wahrnehmbar sein. - Das Gehirn liegt an der Grenze des ersten und zweiten Fünftels. - Der fein gefaltete, krausenförmige $\mathrm{Ph}$ arynx ist dem hintern Ende näher als dem vordern - bei Leptoplaniden ja ein normales Verhalten -, die Länge der Pharyngealtasche beträgt ein Drittel der Körperlänge. Im Verhältniss zum Pharynx ist auch der $\mathrm{Mund}$ noch etwas nach hinten verschoben, so dass er fast ans Ende des zweiten Drittels zu liegen kommt. $5 \mathrm{~mm}$ vom Hinterrand befinden sich dicht hinter einander gelegen, aber getrennt die Geschlechtsöffnungen. 
Da die äussere Untersuchung selbst des aufgehellten Thieres bei Polycladen im Allgemeinen, vor allem aber bei Leptoplaniden meist ganz ungenügende Resultate ergiebt, eine Beschreibung eines nur äusserlich untersuchten Thieres also ziemlich werthlos ist, entschloss ich mich, das einzige vorhandene Exemplar zur Herstellung einer Schnittserie zu opfern, um wenigstens über die Anatomie des Geschlechtsapparats ins Klare zu kommen. Ueber den feinern Bau der Organe vermag ich trotz der Schnitte keine Angaben zu machen. Bedauerlicher Weise war gerade dieses interessante Thier das einzige,

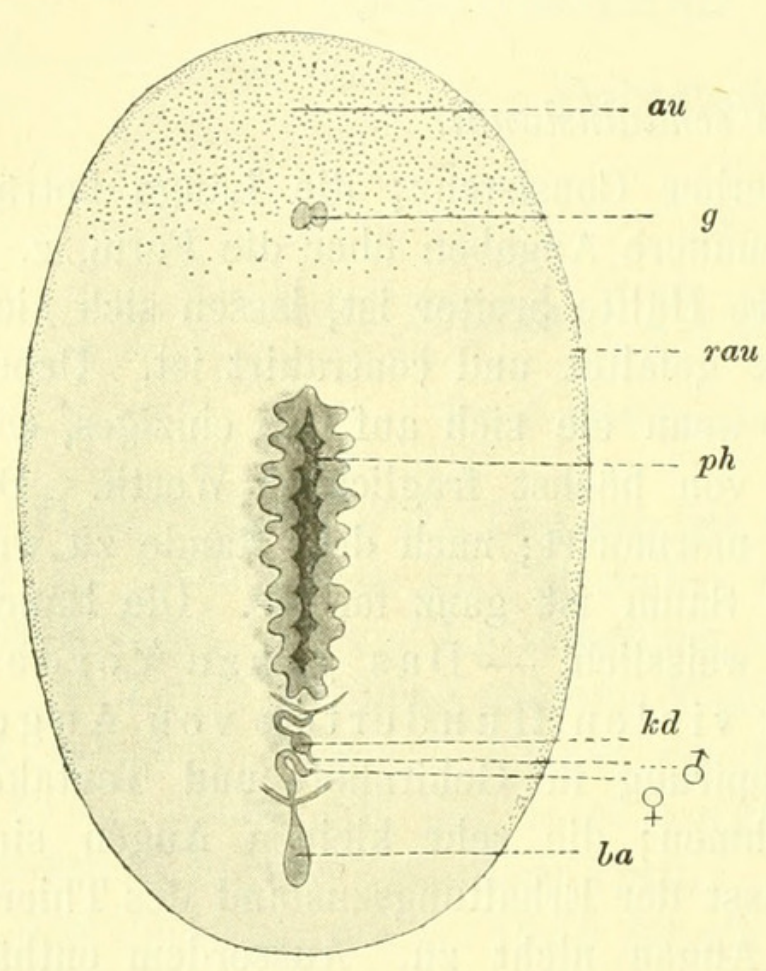

Fig. A. Microcelis schauinslandin. $g . n . s p$. au Augen, ba accessorische Blase, g Gehirn, ghau Gehirnhofaugen, $k d$ Körnerdrüse, $p$ Penis, $p h$ Pharynx, rau Randaugen, tau Tentakelaugen, $\delta$ männliche Oeffnung, $\Varangle$ weibliche Oeffnung.

dessen Conservirungszustand für die histologische Untersuchung nicht völlig genügte. Die Polycladen sind ja in dieser Hinsicht ziemlich unberechenbar.

Die Geschlechtsöffnungen liegen, wie erwähnt, dicht hinter einander am Ende des vierten Fünftels. Der Pen is ist klein, unbewaffnet, nach hinten gerichtet; eine eigentliche Samenblase existirt nicht, dagegen ist ein längeres Stück des Vas deferens mit Musculatur versehen und functionirt als Samenblase; es mündet in eine kleine, kuglige Körnerd r ü se, deren Ausführungsgang direct in den Penis eintritt. Am weiblichen Apparat ist die Schalendrüse mächtig entwickelt; eine sehr grosse acces sorische Blase erreicht fast das Hinterende des Körpers.

Der Bau des Geschlechtsapparats würde für eine Einreihung in das Genus Leptoplana sprechen, die Augenstellung weicht aber bei unserm Thier $\mathrm{zu}$ bedeutend von den übrigen Leptoplana-Arten ab; sie erinnert an Cestoplana, bei welcher auch der vordere Körpertheil mit gleichmässig vertheilten Augen versehen ist; sie sind hier freilich noch sehr viel zahlreicher und finden sich, wie erwähnt, überdies fast am ganzen Rande, ähnlich wie bei Discocelis und Cryptocelis. 
Alle übrigen Polycladen - sie stammen von zwei Fundstellen am French Pass und von dreien auf den Chatham-Inseln gehören zum Genus Leptoplana. Der grössere Theil war zu jung, als dass die Species hätte bestimmt werden können; der Geschlechtsapparat war meist noch gar nicht oder doch nur in der ersten Anlage vorhanden. Da die Thiere in Farbe, Gestalt und Consistenz ziemlich verschieden sind, so würde die Vermuthung nahe liegen, dass sie nicht der gleichen Art angehören; andrerseits variiren aber bekanntlich immer die Vertreter der gleichen Leptoplana-Species recht bedeutend in diesen äussern Eigenschaften und sind die reifen Exemplare, die mir zur Untersuchung vorlagen, trotz grosser äusserer Verschiedenheiten, sicher der gleichen Art angehörig - nach ihrem anatomischen Bau zu urtheilen. Unter diesen Umständen muss die Frage nach der Zusammengehörigkeit der jungen Thiere unentschieden bleiben. Die bestimmbaren Thiere gleichen Leptoplana californica mihi ${ }^{1}$ ), obwohl sie in grösserm oder geringerm Grade einige Abweichungen aufweisen. Dieselben beziehen sich auf der Buchstaben siehe bei Fig. A.

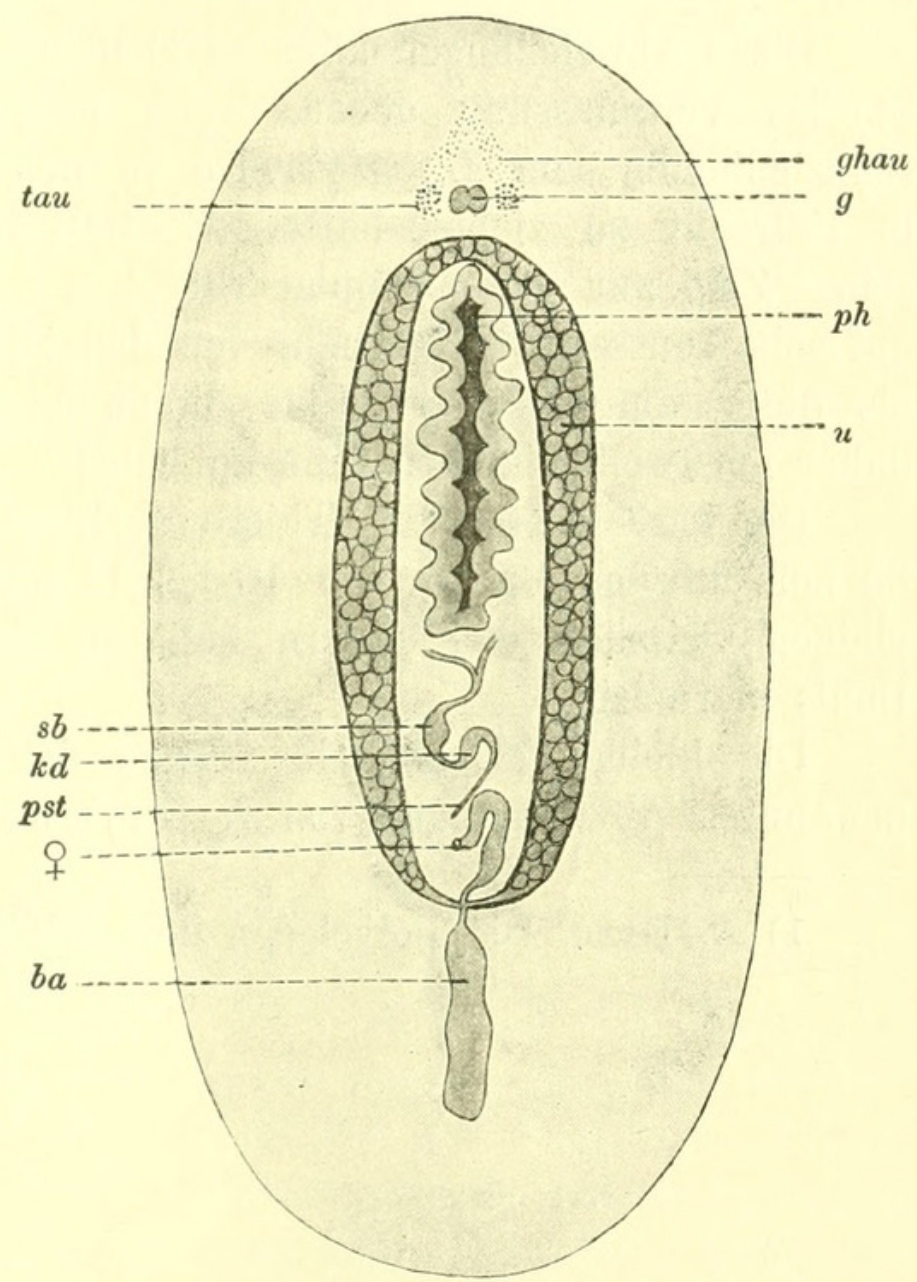

Fig. B. Leptoplana californica (mihi). Erklärung folgende Verhältnisse:

1) die gemeinsame Geschlechtsöffnung ist vom Hinterende noch weiter entfernt als bei $L$. california;

2) die accessorische Blase ist noch beträchtlich grösser;

1) M. Phenn, Drei neue Polycladen, in: Jena. Zeitschr. Na turw. V. $31,1897$. 
3) der Pharynx und in ihm der Mund sind noch weiter nach vorn gelagert, was nach $\mathrm{LANG}^{1}$ ) für eine Leptoplanide eigentlich ungehörig ist; eine Tendenz zur Verlagerung nach hinten ist da im Allgemeinen eher zu erwarten;

4) Die beiden charaktèristischen grossen Augen, mit denen bei L. californica die Gruppen der Gehirnhofaugen vorn abschliessen, fehlen hier; statt dessen rücken bei manchen Exemplaren - eine völlige Uebereinstimmung ist nicht vorhanden - vereinzelte Augen noch bedeutend weiter nach vorn.

Diese Abweichungen könnten vielleicht zur Gründung einer neuen Species Veranlassung geben, wenn man einige constante Verschiedenheiten von $L$. californica in den äussern Eigenschaften mit in Betracht zu ziehen hätte. Es hat mir nun von $L$. californica seiner Zeit nur ein Exemplar vorgelegen, und dasselbe ist in Bezug auf sein Aeusseres nicht mehr von den jetzt untersuchten verschieden als diese von einander. Eine Trennung in zwei Arten scheint mir daher einstweilen keinen Sinn zu haben.

Die geschlechtsreifen Thiere variiren in der Länge von 20 bis $35 \mathrm{~mm}$, in der Breite von 10 bis $16 \mathrm{~mm}$. Die Rückenseite ist bei einigen weisslich, bei andern hell- bis dunkelbraun, theils einfarbig, theils marmorirt.

Die kleine schematische Skizze der Anatomie lässt die oben erwähnten Abweichungen von $L$. californica deutlich erkennen.

1) A. Lang, Die Polycladen des Golfes von Neapel, 1884. 


\section{$2 \mathrm{BHL}$ Biodiversity Heritage Library}

1899. "Ergebnisse einer Reise nach dem Pacific (Schauinsland, 1896 \& 1897), Polycladen." Zoologische Jahrbücher 12, 448-452.

https://doi.org/10.5962/bhl.part.2035.

View This Item Online: $\underline{\text { https://www.biodiversitylibrary.org/item/38879 }}$

DOI: https://doi.org/10.5962/bhl.part.2035

Permalink: https://www.biodiversitylibrary.org/partpdf/2035

\section{Holding Institution}

MBLWHOI Library

Sponsored by

MBLWHOI Library

\section{Copyright \& Reuse}

Copyright Status: NOT_IN_COPYRIGHT

This document was created from content at the Biodiversity Heritage Library, the world's largest open access digital library for biodiversity literature and archives. Visit BHL at https://www.biodiversitylibrary.org. 